\title{
NOUVELLE
}

\section{CDX2 : un facteur à multiples facettes pour les cellules épithéliales du tube digestif}

François Boudreau, Nathalie Rivard, Fernand-Pierre Gendron, Jean-François Beaulieu
Département d'anatomie et de biologie cellulaire, Faculté de médecine et des sciences de la santé, Chaire de recherche du Canada

en physiopathologie intestinale, Université de Sherbrooke, $12^{\mathrm{e}}$ Avenue Nord, Sherbrooke, Sherbrooke (Québec), JlH 5N4 Canada.

Jean-Francois.Beaulieu@USherbrooke.ca
> L'épithélium intestinal est en constant renouvellement grâce à l'établissement d'un équilibre entre la prolifération et la différenciation cellulaires. De nombreuses pathologies de l'épithélium intestinal, en l'occurrence le cancer colorectal, découlent directement du dérèglement des mécanismes régissant cet équilibre. L'utilisation de modèles cellulaires et de souris transgéniques a montré l'importance positionnelle et combinatoire de certains facteurs de transcription dans la régulation de marqueurs associés à la différenciation épithéliale tout le long du tube digestif $[1,2]$. CDX2, un facteur de transcription exprimé spécifiquement dans l'épithélium intestinal, représente un candidat important dans la mise en œuvre d'un programme spécifique de différenciation temporelle en fonction des segments de l'épithélium du tube digestif. Certaines études utilisant des modèles de souris génétiquement modifiées pour exprimer de façon ectopique CDX2 dans la muqueuse gastrique ont démontré son rôle déclencheur dans le développement de métaplasies intestinales [3].

II a été montré récemment que CDX2, présent dans les tumeurs de l'œsophage et de l'estomac, module de façon positive le gène de la guanylyle cyclase $C(G C-C)$, un suppresseur de tumeurs dont l'expression est normalement restreinte à l'épithélium intestinal [4]. La présence anormale de CDX2 dans les pathologies du tube digestif antérieur était déjà connue. L'originalité de l'étude en question vérifie un concept moléculaire crucial sur la régionalisation des fonctions de l'épithélium le long du tube digestif en situation physiopathologique. En effet, le gène $G C-C$ code un récepteur transmembranaire sensible aux entérotoxines bactériennes responsables de syndromes diarrhéiques qui affecte, entre autres, la prolifération cellulaire de l'épithélium intestinal. Le patron d'expression de GC-C est parfaitement parallèle à l'expression de CDX2 dans les adénocarcinomes de l'œsophage et de l'estomac. Ainsi, cette étude concilie au niveau moléculaire la conséquence directe d'un gain anormal de l'expression du facteur de transcription CDX2 dans des pathologies du tube digestif antérieur avec l'apparition de l'expression de gènes normalement restreints à l'intestin. En utilisant une lignée d'adénocarcinome d'œsophage humain, l'étude propose enfin une relation signalétique entre une concentration élevée d'acides biliaires, l'activation du facteur nucléaire $\kappa B(N F-\kappa B)$ et la régulation transcriptionnelle du gène CDX2. Malgré l'établissement d'une telle corrélation moléculaire, la question perdure sur la nature des mécanismes cellulaires qui dépendent d'une expression aberrante de CDX2 dans le contexte tumoral. Nos travaux ont récemment contribué à une meilleure compréhension de ces mécanismes.
En effet, notre groupe a mis en évidence plusieurs caractéristiques de CDX2 dans le contrôle de la fonction épithéliale intestinale. CDX2 était déjà connu comme régulateur de la prolifération et de la différenciation épithéliale intestinale de cellules de rongeurs en culture [5]. Plus récemment, l'introduction de CDX2 dans la lignée épithéliale intestinale humaine normale HIEC nous a permis non seulement de confirmer son rôle antiprolifératif impliquant une diminution des niveaux de la cycline $D 1$, une protéine régulatrice du cycle cellulaire, mais aussi de démontrer que son action sur la différenciation intestinale nécessitait la présence de régulateurs supplémentaires absents dans cette lignée humaine normale [6].

À l'inverse, nous avons montré que l'expression de CDX2 est modulée durant la progression du cycle cellulaire. En effet, CDX2 est capable d'interagir avec la kinase associée aux cyclines, Cdk2, de même qu'avec l'inhibiteur du cycle cellulaire p27 $7^{\mathrm{Kipl}}$ [7]. Dans les cellules prolifératives, les complexes cycline $\varepsilon / C d k 2$ et cycline $A / C d k 2$, responsables respectivement de l'entrée et de la progression de la phase S, phosphorylent CDX2 (et p $27^{K i p l}$ ), ce qui entraîne sa sortie du noyau et sa dégradation dans le protéasome. L'activité de CDX2 peut aussi être modulée par des modifications post-traductionnelles telles que la phosphorylation. CDX2 est spécifiquement phosphorylé sur le résidu sérine 
en position 60 dans le compartiment prolifératif de la crypte intestinale [8]. Cette phosphorylation se traduit par une activité transcriptionelle moindre et dépend de l'activité des MAP-kinases ERK1/2. Par ailleurs, la MAP-kinase $p 38 \alpha$ peut aussi phosphoryler CDX2 dans un contexte de différenciation cellulaire et ainsi stimuler son activité transcriptionnelle [9] (Figure I).

L'identification de plusieurs gènes cibles de CDX2 a été rapportée au cours des dernières années. Ces gènes présentent des fonctions associées à la prolifération, à la différenciation, à la polarisation et aux fonctions métaboliques de l'entérocyte. Parmi ceuxci, la furine, une convertase impliquée dans la maturation protéolytique de proprotéines, représente une nouvelle classe de molécules potentiellement impliquées dans la différenciation épithéliale de l'intestin. En effet, en augmentant l'expression de la furine, CDX2 contrôlerait la maturation posttraductionnelle de protéines membranaires telles que la $\varepsilon$-cadhérine

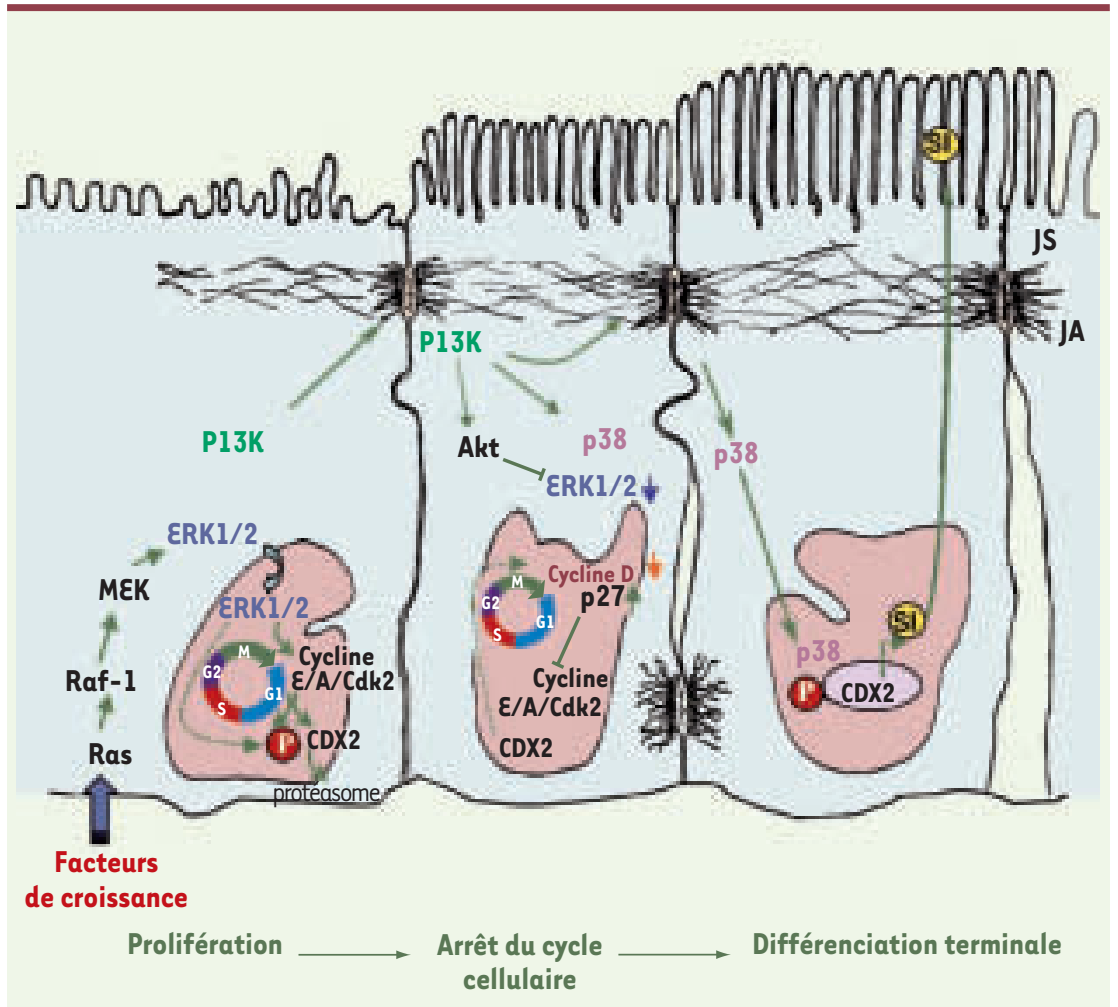

Figure 1. Voies de signalisation impliquant CDX2. Dans les cellules indifférenciées, les complexes cyclines $\varepsilon / A / C d k 2$ activent la progression du cycle cellulaire et inhibent la différenciation en phosphorylant CDX2, induisant sa dégradation dans le protéasome cytoplasmique. Lorsque les cellules sont induites à se différencier, l'engagement des cadhérines provoque le recrutement et l'activation de la PI3 kinase (PI3K) qui à son tour, active la kinase Akt et induit l'inhibition des $\varepsilon R K 1 / 2$, ce qui augmente l'expression de $p 27^{\text {kipl }}$ qui inhibe l'activité des complexes cyclines $\varepsilon / A$ / Cdk2 et le cycle cellulaire en phase G1. ERK1/2 modifie le niveau de phosphorylation de CDX2 de façon directe ou indirecte (ligne pointillée). Une surexpression de CDX2 mène à une diminution de la cycline D. La PI3K stimule aussi l'organisation du cytosquelette et la maturation des jonctions, ce qui stimule la p38MAP-kinase (isoforme $\alpha$ ) qui se rend au noyau où elle phosphoryle CDX2, augmentant son activité transcriptionnelle, favorisant ainsi la différenciation. PI3K : phosphatidylinositol 3-kinase ; JS : jonctions serrées ; JA : jonctions adhérentes; SI : sucrase-isomaltase ; $\mathrm{P}$ : phosphorylation.

et la lactase-phlorizine hydrolase, ellesmêmes impliquées dans la différenciation entérocytaire [10].

Les progrès des dernières années relatifs à l'identification des mécanismes clés de la régulation positionnelle, de la modification post-traductionelle et de la stabilité du facteur CDX2 en fonction de la signalisation intracellulaire auront permis d'amorcer la démystification du rôle de ce régulateur puissant dans le contexte des pathologies tumorales du tube digestif. Le défi des prochaines années sera sans aucun doute d'exploiter ces découvertes pour établir de nouvelles stratégies diagnostiques et thérapeutiques ciblées sur les désordres pathologiques de l'épithélium du tube digestif. $\diamond$

CDX2: a transcription factor

with multiple tasks for epithelial

cells of the digestive tract

\section{RÉFÉRENCES}

1. Boudreau F, Rings EH, Van Wering HM, et al. Hepatocyte nuclear factor-1 alpha, GATA-4, and caudal related homeodomain protein $\mathrm{Cdx} 2$ interact functionally to modulate intestinal gene transcription. Implication for the developmental regulation of the sucrase-isomaltase gene. J Biol Chem 2002; 277 : 31909-17

2. Boudreau F, Rings $\varepsilon H$, Swain GP, et al. A novel colonic repressor element regulates intestinal gene expression by interacting with Cux/CDP. Mol Cell Biol 2002 ; 22 : 5467-78.

3. Silberg DG, Sullivan J, Kang $\varepsilon$, et al. Cdx2 ectopic expression induces gastric intestinal metaplasia in transgenic mice. Gastroenterology 2002; 122 : 689-96.

4. Debruyne PR, Witek M, Gong L, et al. Bile acids induce ectopic expression of intestinal guanylyl cyclase $C$ through nuclear factor-kappaB and $\mathrm{Cd} \times 2$ in human esophageal cells. Gastroenterology 2006 ; 130 : 1191-206.

5. Suh $\varepsilon$, Traber PG. An intestine-specific homeobox gene regulates proliferation and differentiation. Mol Cell Biol $1996 ; 16: 619-25$.

6. Escaffit F, Pare F, Gauthier R, et al. Cdx2 modulates proliferation in normal human intestinal epithelial crypt cells. Biochem Biophys Res Commun 2006; 342 : 66-72.

7. Boulanger J, Vézina A, Mongrain S, et al. Cdk2-dependent phosphorylation of homeobox transcription factor CDX2 regulates its nuclear translocation and proteasomemediated degradation in human intestinal epithelial cells. J Biol Chem 2005 ; 280 : 18095-107.

8. Rings $\varepsilon H$, Boudreau F, Taylor JK, et al. Phosphorylation of the serine 60 residue within the $C \mathrm{dx} 2$ activation domain mediates its transactivation capacity. Gastroenterology $2001 ; 121: 1437-50$.

9. Houde M, Laprise P, Jean D, et al. Intestinal epithelial cell differentiation involves activation of $p 38$ mitogenactivated protein kinase that regulates the homeobox transcription factor CDX2. J Biol Chem 2001; $276: 21885-94$

10. Gendron FP, Mongrain S, Laprise P, et al. The CDX2 transcription factor regulates furin expression during intestinal epithelial cell differentiation. Am J Physiol Gastrointest Liver Physiol 2006 ; 290 : G310-8. 